\title{
Overview of recombinant human hyaluronidase-facilitated subcutaneous infusion of IgG in primary immunodeficiencies
}

\begin{abstract}
Subcutaneous administration of immunoglobulin (IGSC) in a home setting, compared with intravenous administration, can improve patient quality of life. During IGSC, however, the subcutaneous extracellular matrix inhibits flow and fluid entry into the vascular compartment, which limits the amount of drug delivered. Recombinant human hyaluronidase ( $\mathrm{rHuPH} 20$ ) increases the absorption and dispersion of infused fluids and drugs. Results from a Phase III, prospective, open-label, noncontrolled study of patients with primary immunodeficiencies indicated that IGSC infusion, facilitated by rHuPH20, is well tolerated and delivers infusion volumes at treatment intervals and rates equivalent to intravenous administration. This drug evaluation provides an overview of rHuPH2O and results of clinical studies of IGSC infusion facilitated by rHuPH20 in patients with primary immunodeficiencies.
\end{abstract}

Keywords: facilitated subcutaneous infusion $\bullet$ hyaluronidase $\bullet \lg G$ • pharmacokinetics

- Phase III • primary immunodeficiency disease $\bullet$ recombinant human hyaluronidase $\bullet$ safety

- tolerability

\section{Background}

Primary immunodeficiencies (PIDs) are a heterogeneous group of disorders characterized by increased susceptibility to infection. The most common PID phenotype is defective antibody production that may be complete or partial, rendering patients susceptible to severe, recurrent pyogenic infections [1]. In 2011, approximately 6 million people worldwide were estimated to be living with a PID. The actual prevalence of PID, however, is unknown. A recent analysis of national registries and the Jeffrey Modell Centers Network indicated that only 27,000-60,000 patients are estimated to be diagnosed with the disorder to date [2]. An earlier phone survey in the USA indicated that as many as 150,000-360,000 persons are diagnosed with a PID [3]. Regardless, many patients with PID are believed to be undiagnosed and untreated [2].

The cornerstone of treatment for antibody production defects is immunoglobulin replacement with purified IgG. Owing to improvements in IgG processing, intravenous (iv.) administration of IgG (IGIV) has been the standard of care in the USA and in much of the world for more than 30 years [4,5]. IGIV treatment, however, can be burdensome to patients, as it requires iv. access and monitoring by a healthcare professional. IGIV has also been associated with a significant rate of systemic adverse reactions, including nausea, headache and myalgia, as well as more significant reactions such as renal failure, thromboembolic events, aseptic meningitis and rare hypersensitivity reactions [6].

Given the challenges associated with IGIV, sc. administration of IgG (IGSC) has emerged as an increasingly used alternate. The availability of an IgG product for sc. use in 2006 increased interest in the use of IGSC for patients with PID. IGSC therapy is an appropriate choice for many patients with PID who have difficulty with venous access or who do not tolerate iv. infusions, or for patients or caregivers who desire a better quality of life in a home setting [7-15]. IGSC
Richard L Wasserman

DallasAllergyImmunology, 7777 Forest Lane, Suite B-332, Dallas, TX 75230, USA Tel.: +1972 5667788

Fax: +19725668837

drrichwasserman@gmail.com 
has been shown to be as effective as IGIV, with fewer systemic reactions than IGIV [5,6], which is probably due to more frequent, small infusions and slow absorption of IGSC into the vascular compartment. Infusion of smaller volumes of IgG at shorter intervals through the sc. route appears to decrease the frequency of systemic reactions [16]. Patients who have experienced difficulties with venous access or who cannot tolerate IGIV infusions are also likely to benefit from IGSC [17].

Standard IGSC is not without limitations. Hyaluronan (also called 'hyaluronic acid') in the extracellular matrix (ECM), a key component of sc. tissue, impedes the flow of fluids introduced into the sc. space and plays a major role in the movement of fluids that ultimately enter the vascular compartment (Figure 1) [18]. Because hyaluronan limits the flow of fluid, the amount of drug that can be delivered subcutaneously is markedly less than the amount of drug that can be delivered during IGIV infusions. The limited fluid flow during sc. infusion can also trap some drugs in the ECM, potentially intensifying infusion-site reactions and reducing bioavailability [18].

Several strategies have been developed to overcome the limitations imposed on IGSC by the ECM. One approach is to increase the concentration of $\operatorname{IgG}$ in a sc. formulation. While the use of more concentrated formulations can reduce the volume needed, the viscosity, solubility and aggregation potential of proteins, along with the occasional local irritation caused by the delivery of proteins, limit the concentration of drug that can be infused [18]. Another strategy is to increase the rate of IGSC administration by increasing the number of infusion sites. For therapeutic agents that require large volumes owing to dose or limited solubility, it is often necessary to administer small-volume doses more frequently and/ or to use multiple infusion sites.

A more recent approach is IGSC administration that is facilitated by preinfusing recombinant human hyaluronidase ( $\mathrm{rHuPH} 20$; Halozyme Therapeutics Inc., CA, USA; rHuPH20-facilitated subcutaneous infusion of IgG [IGHy; HyQvia; Baxter Innovations $\mathrm{GmbH}$, Vienna, Austria]). This infusion method has been shown to enhance the delivery of IgG to patients with PID [16]. In this article, $\mathrm{rHuPH} 20$ as a facilitator of sc. infusions and IGSC, and the results of clinical trials of IGHy are reviewed.

\section{Hyaluronidase}

Hyaluronidase enzymatic activity was first identified by Duran-Reynals in the 1920s as a spreading factor in extracts from rabbit testes and was subsequently isolated from vitreous humor and other tissues $[19,20]$. Hyaluronidase was named for its ability to digest hyaluronan (Figure 2). Following its discovery,

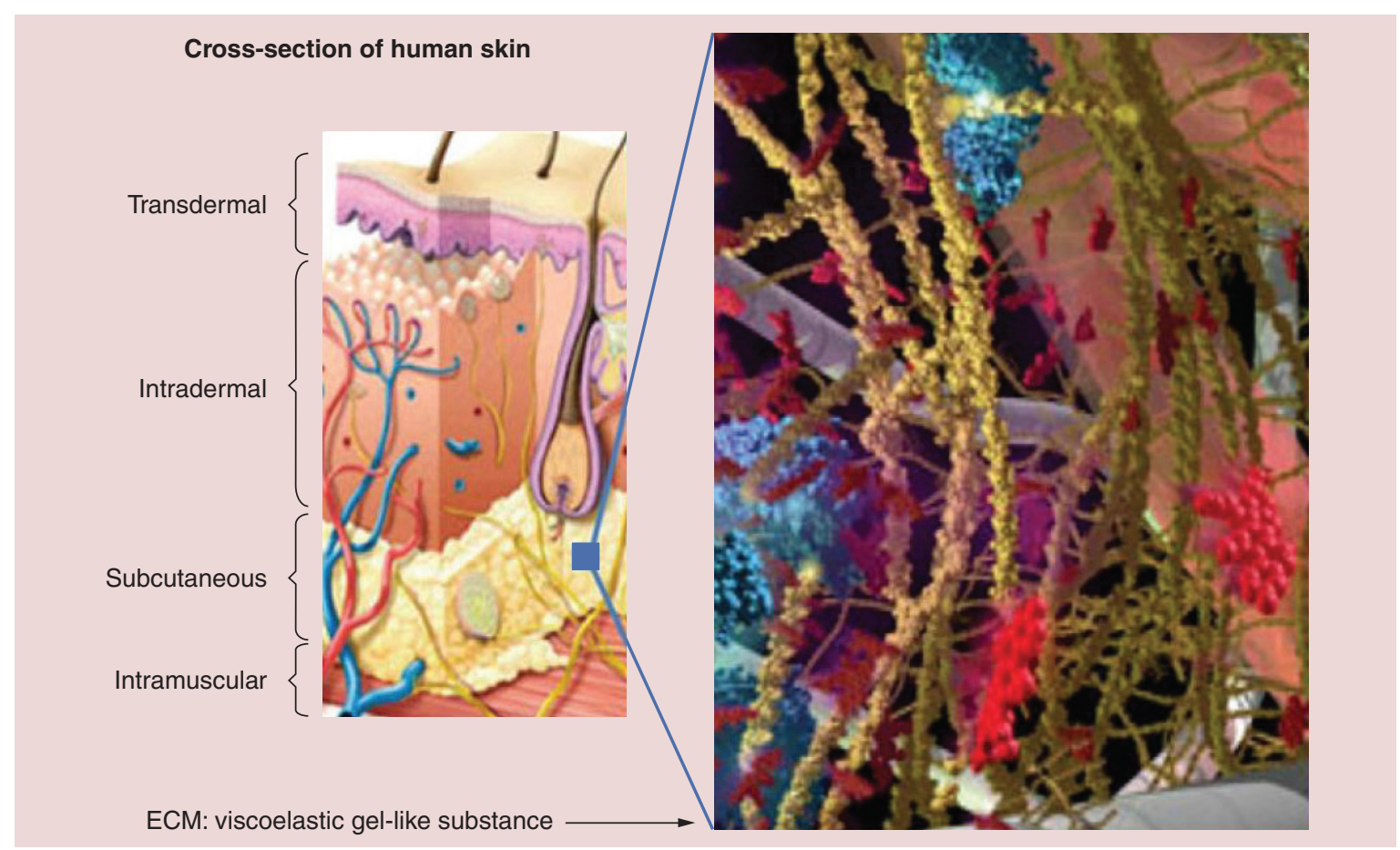

Figure 1. Subcutaneous administration barriers. Rate and volume of subcutaneous administration is limited by the flow restrictions caused by hyaluronan in the ECM, which can be temporarily modified with the injection of hyaluronidase, facilitating the dispersion of drug molecules.

ECM: Extracellular matrix.

Reproduced with kind permission from [4] Springer Science and Business Media. 

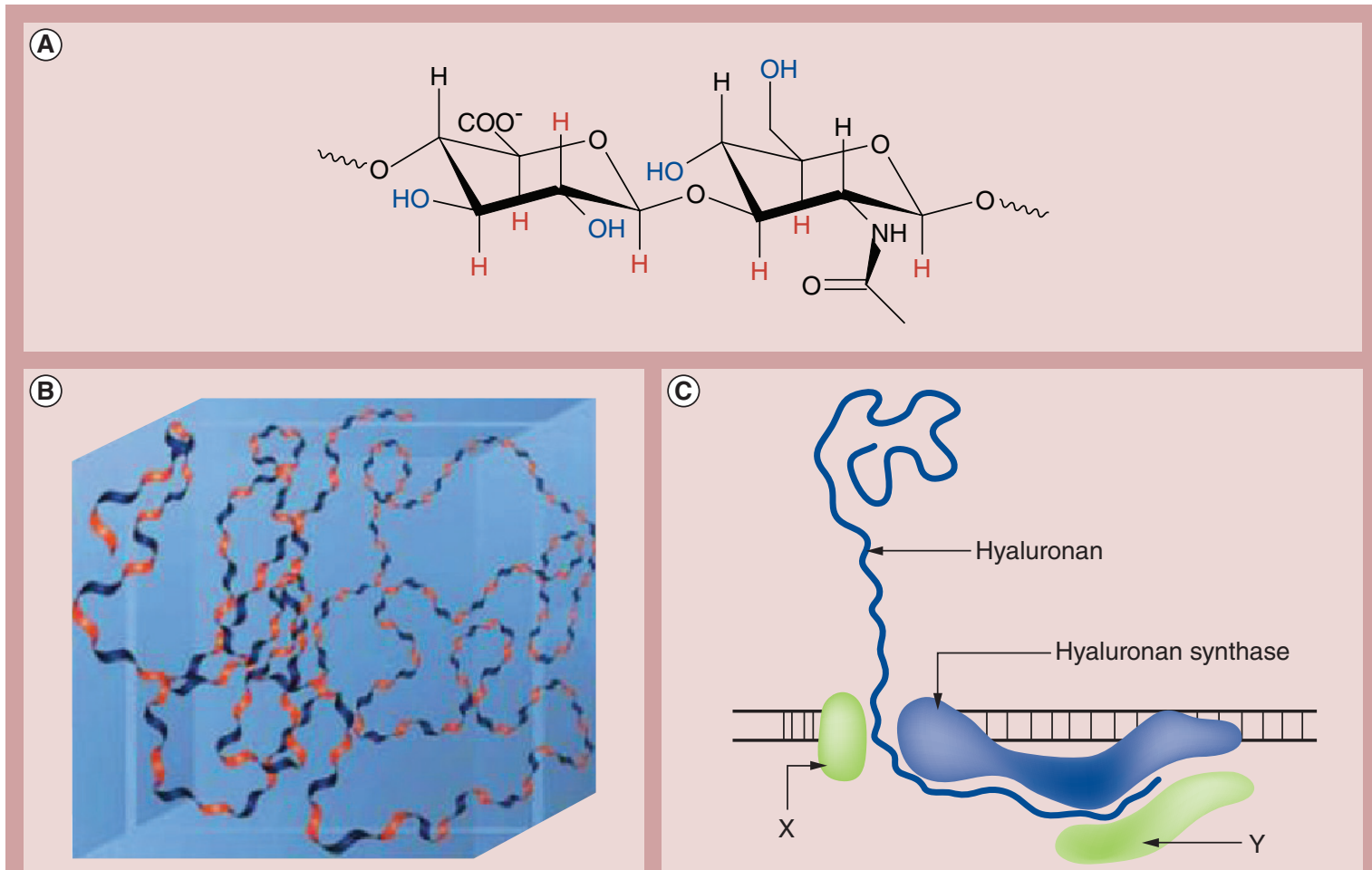

Figure 2. Structure and synthesis of hyaluronan. Hyaluronan forms large, random coil structures, binds large quantities of water and restricts flow through the extracellular matrix. (A) The structure of hyaluronan, which comprises repeating disaccharides of glucuronic acid and $\mathrm{N}$-acetylglucosamine. Reprinted with permission from Macmillan Publishers Ltd on behalf of Cancer Research UK [21]. (B) The expansion of hyaluronan molecules in dilute solutions, thereby allowing for the occupation of large volumes with trapped water inside the structure. Reproduced with permission [22]. (C) Hyaluronan synthesis at the inner surface of the plasma membrane; its close relationship with the cell surface allows for ready participation in the creation of a pericellular hydrated zone. Putative regulatory proteins are indicated by $\mathrm{X}$ and $\mathrm{Y}$.

Reproduced with permission from [23].

fractionated extracts from bovine testes with demonstrated hyaluronidase activity were developed as early pharmaceutical forms of the product.

The identification of the human hyaluronidase PH20 gene [24] more than 70 years after the discovery of hyaluronidase, led to the development of $\mathrm{rHuPH} 20$ [18]. Today, ovine [25], bovine [26] and human recombinant [27] preparations of hyaluronidase are used to increase the absorption and dispersion of drugs injected subcutaneously [28]; however, the animalderived preparations are contaminated with testicular proteins and are immunogenic and thus not intended for long-term use. Only the rHuPH20 component of IGHy has been clinically demonstrated to be safe and effective in enhancing the sc. delivery of IgG [29]. The mechanism of action of rHuPH20 is to transiently and locally degrade the gel-like hyaluronan present in the ECM, decreasing both the viscosity of the hyaluronan and the resistance to fluid flow in the local sc. space, thereby allowing increased dispersion of the $\mathrm{IgG}$ and enabling conversion of drug therapies from iv. to sc. administration.
The enzymatic activity of $\mathrm{rHuPH} 20$ is highly specific for the $\beta 1-4$ linkage in glycosaminoglycans. There is no enzymatic activity of $\mathrm{rHuPH} 20$ against $\mathrm{N}$-linked glycans or polypeptide backbones of proteins, and $\mathrm{rHuPH} 20$ has no effect on the structure or properties of IgG, including the glycosylated side chains.

According to the US FDA, hyaluronidase products are distinct entities from one another owing to the variations in chemical composition and source material. Therefore, the products are similar but not interchangeable and have been approved for the dispersion and absorption of other drugs and fluids [28]. rHuPH20, the only FDA-approved recombinant human hyaluronidase, is indicated for use as an adjuvant to enhance absorption and dispersion of drugs and fluids administered subcutaneously (Figure 3) [27].

Animal hyaluronidase extracts are not as pure as rHuPH20, as these extracts typically contain approximately $15 \%$ of the specific activity per milligram of total protein compared with rHuPH20 (>100,000 USP 

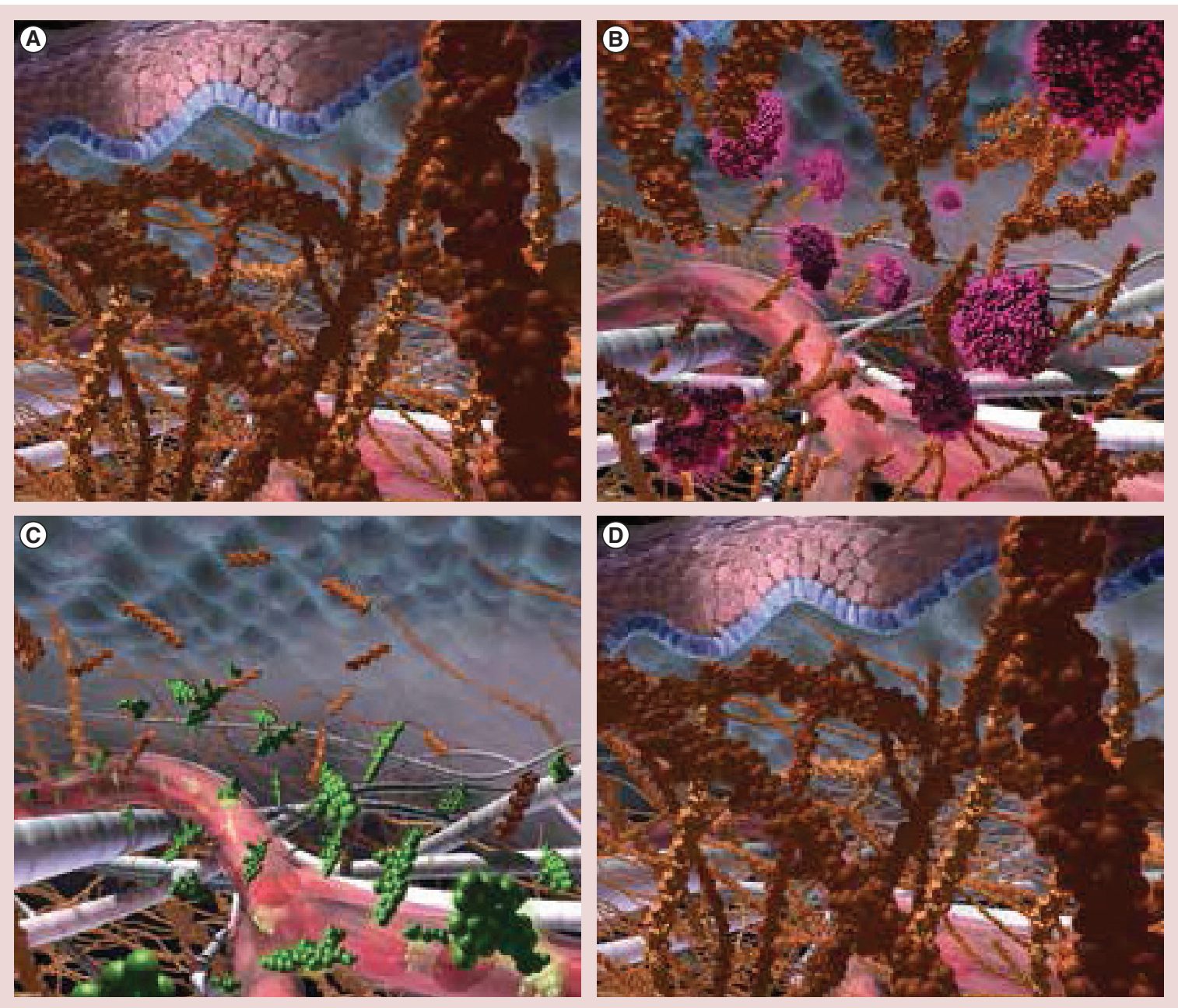

Figure 3. Proposed recombinant human hyaluronidase mechanism of action in the subcutaneous space. In this depiction of a cross-sectional view of skin dermis, (A) the hyaluronan matrix (gold) creates resistance to bulk fluid. (B) Recombinant human hyaluronidase (purple) decreases local resistance to injection of other molecules, (C) allowing exposure of these molecules (green) to capillaries and lymphatics. (D) The hyaluronan barrier is rapidly reconstituted.

Reproduced with permission from Halozyme Therapeutics [30].

units/mg protein) [31,32]. In addition, animal-derived hyaluronidase products are often immunogenic in humans because the hyaluronidase itself is a xenogeneic protein and may be contaminated with additional nonhuman proteins, including proteases, immunoglobulins, anticoagulants, growth factors, vasopermeability factors and other impurities [18,32]. These animal-derived hyaluronidase preparations can elicit $\mathrm{IgE}$-mediated allergic reactions after repeated administration [33-35].

\section{Recombinant human hyaluronidase}

Preclinical studies to evaluate the safety of rHuPH20

Preclinical studies have shown that $\mathrm{rHuPH} 20$ is fast acting with a local half-life in the sc. space of less than $30 \mathrm{~min}$ (rodent model) [36], and is detectable in the blood only at the 1-min time point following iv. administration in rats [18].

The safety of rHuPH20 administered by sc. injection has been evaluated in primates. $\mathrm{rHuPH} 20$ was well tolerated at 45,000 units/injection by sc. infusion ( $>9000$-times the typical dose given with IGHy) [18]. No clinically detectable inflammatory reactions or histologically identifiable inflammatory cell infiltrates were seen when infusion sites were compared with the carrier control in animals sampled $24 \mathrm{~h}$ to 28 days postinjection.

In swine studies, Kang et al. evaluated the potential impact of large volume sc. infusion on the local tissue [37]. Data demonstrated that, compared with a control infusion, rHuPH20 significantly improved $\mathrm{IgG}$ dispersion - including reduced mean local swelling volume $\left(250 \mathrm{~mm}^{2}\right.$ with placebo vs $32 \mathrm{~mm}^{2}$ 
with rHuPH20; $<0.0001)$ and area $\left(150 \mathrm{~mm}^{2}\right.$ with placebo vs $62 \mathrm{~mm}^{2}$ with rHuPH20; $\left.<<0.0001\right)$, as well as a reduced mean inline pressure associated with IgG infusion (128 mmHg with placebo vs $199 \mathrm{mmHg}$ with rHuPH20; $<$ 0.0001) - while minimizing local tissue damage. These data indicated that $\mathrm{rHuPH} 20$ could enable sc. administration of large IgG volumes $(300 \mathrm{ml})$ at a single site.

\section{Clinical studies using rHuPH20 to facilitate sc. drug delivery}

The safety and efficacy of rHuPH20 to enhance dispersion and absorption of fluids and drugs administered subcutaneously were demonstrated in several clinical studies [38-40].

$\mathrm{rHuPH} 20$ is being investigated in a number of therapeutic areas, including endocrinology, immunology and oncology. Table 1 details various product combinations with $\mathrm{rHuPH} 20$ that are currently in development. Several investigational trials with $\mathrm{rHuPH} 20$ are described in the following sections.

\section{Narcotic analgesia}

The Increased Flow Utilizing Subcutaneously Enabled Morphine Phase III study (Clinicaltrials.gov identifier: NCT00593281) was a randomized, placebo-controlled trial involving 13 patients in a hospice or palliative care setting [40]. A three-way crossover design was employed using morphine at a dose of $5 \mathrm{mg}$, with each patient serving as his or her own control, and the primary end point was the $T_{\text {max }}$ for morphine. Morphine in combination with rHuPH20 administered subcutaneously enhanced the absorption rate of morphine compared with sc. morphine plus placebo, significantly reducing the mean $\mathrm{T}_{\max }$ from 13.8 to $9.2 \mathrm{~min}$, a decrease of $33 \%$ $(p=0.026)$. The majority of adverse events (AEs) were local, mild and transient. No non-infusion-site events were associated with $\mathrm{rHuPH} 20$. Although this study was not designed to assess efficacy, sc. morphine in combination with $\mathrm{rHuPH} 20$ provided pharmacokinetic $(\mathrm{PK})$ characteristics that were superior to those of sc. morphine plus placebo.

\section{Case study of hyaluronidase (ovine)-facilitated IGSC self-administration}

Promising data derived from treatment of a 28 -yearold female patient with common variable immunodeficiency disease provided proof of concept for selfadministered hyaluronidase-facilitated IGSC [42]. This patient presented with low serum IgG levels $(1.99 \mathrm{~g} / \mathrm{l})$ and an inability to make specific antibodies, and had poor venous access and an inability to achieve adequate

Table 1. Selected approved and investigational uses of recombinant human hyaluronidase.

\begin{tabular}{|c|c|c|c|c|}
\hline \multicolumn{2}{|c|}{ Products and product candidates } & \multirow{2}{*}{$\begin{array}{l}\text { Therapeutic } \\
\text { area }\end{array}$} & \multirow[t]{2}{*}{ Use/indication } & \multirow{2}{*}{$\begin{array}{l}\text { Development } \\
\text { stage }\end{array}$} \\
\hline rHuPH20 \pm product & Product name & & & \\
\hline rHuPH20 & 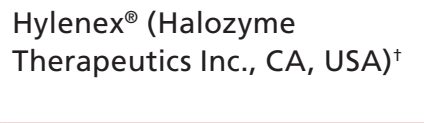 & $\begin{array}{l}\text { Various; } \\
\text { endocrinology }\end{array}$ & $\begin{array}{l}\text { Peptide, small } \\
\text { molecule and fluid } \\
\text { delivery }\end{array}$ & $\begin{array}{l}\text { Approved } \\
\text { (US FDA) }\end{array}$ \\
\hline $\begin{array}{l}\text { rHuPH} 20+\operatorname{lgG} 10 \%{ }^{\ddagger} \\
(\text { IGHy) }\end{array}$ & $\begin{array}{l}\text { HyQvia (Baxter Innovations } \\
\text { GmbH, Vienna, Austria) }\end{array}$ & Immunology & $\begin{array}{l}\text { Primary } \\
\text { immunodeficiency }\end{array}$ & $\begin{array}{l}\text { Approved } \\
\text { (EMA); } \\
\text { Phase III } \\
\text { (FDA) }\end{array}$ \\
\hline $\begin{array}{l}\text { rHuPH20 + } \\
\text { trastuzumab }\end{array}$ & $\begin{array}{l}\text { Herceptin SC (Roche Pharma } \\
\text { AG, Grenzach-Wyhlen, } \\
\text { Germany) }\end{array}$ & Oncology & Breast cancer & $\begin{array}{l}\text { Approved } \\
\text { (EMA) }\end{array}$ \\
\hline rHuPH20 + rituximab & $\begin{array}{l}\text { MabThera SC (Roche } \\
\text { Products, Welwyn Garden } \\
\text { City, UK) }\end{array}$ & Oncology & $\begin{array}{l}\text { Non-Hodgkin's } \\
\text { lymphoma }\end{array}$ & $\begin{array}{l}\text { Approved for } \\
\text { MabThera } \\
\text { (EMA) }\end{array}$ \\
\hline rHuPH20 + insulin & Analog Insulin-PH2O & Endocrinology & Diabetes & Phase II \\
\hline PEGylated rHuPH20\# & $\begin{array}{l}\text { PEGPH20 (Halozyme } \\
\text { Therapeutics Inc.) }\end{array}$ & Oncology & Solid tumors & Phase II \\
\hline \multicolumn{5}{|c|}{$\begin{array}{l}\text { 'Hylenex }{ }^{\circledast} \text { recombinant (hyaluronidase human injection; Halozyme Therapeutics, Inc., CA, USA). } \\
\text { "Gammagard }{ }^{\circledast} \text { Liquid (immune globulin infusion [human] 10\%; Baxter Healthcare Corporation, CA, USA). } \\
\text { "Herceptin (trastuzumab) } 150 \mathrm{mg} \text { powder for concentrate for solution for infusion (Roche Pharma AG, Grenzach-Wyhlen, Germany). } \\
\text { "MabThera (rituximab) } 100 \mathrm{mg} \text { concentrate for solution for infusion (Roche Products, Welwyn Garden City, UK). } \\
\text { "PEGPH2O increases the half-life of rHuPH20 in the blood, allowing for intravenous administration [41]. } \\
\text { IGHy: Recombinant human hyaluronidase-facilitated subcutaneous infusion of IgG; PEGPH2O: PEGylated recombinant human hyaluronidase; } \\
\text { rHuPH20: Recombinant human hyaluronidase. } \\
\text { Adapted from Halozyme pipeline [41]. }\end{array}$} \\
\hline
\end{tabular}


trough serum IgG levels following the administration of $100 \mathrm{ml}$ Ig via four sc. sites weekly. Self-administration of ovine hyaluronidase (initial dose $150 \mathrm{U} / \mathrm{g}$ of $\mathrm{IgG}$ ) was directly followed by administration of a $16 \%$ IgG sc. formulation (Subcuvia $160 \mathrm{~g} / \mathrm{l}$ solution for infusion; Baxter AG, Vienna, Austria) [42,43]. To compare IGSC with or without hyaluronidase facilitation, IGSC alone was infused into one thigh, and hyaluronidase-facilitated IGSC was infused into the other thigh. Sites on both thighs were then photographed. The IgG dose and infusion rate were increased weekly (loading period 16, 32, 20.8 and $41.6 \mathrm{~g}$ ) until the desired trough level was achieved. An ongoing dose of $20.8 \mathrm{~g}$ $(130 \mathrm{ml})$ of $\mathrm{IgG}$ was given in each site at $100 \mathrm{ml} / \mathrm{h}$; the patient elected to use two sites (both thighs) every 2 weeks. This regimen was developed owing to the lack of swelling at the site where the hyaluronidasefacilitated sc. IgG was administered. Overall, ovine hyaluronidase-facilitated administration reduced the number of infusions, needle sticks and sites needed per month by fourfold (from 16 to 4), while enabling a dose increase from 64 to $84 \mathrm{~g}$ to achieve therapeutic trough levels.

\section{Clinical studies using rHuPH20 to facilitate IGSC infusion \\ Phase I/II study}

A Phase I/II pilot study evaluated the effect of IGHy infusion on the bioavailability of $\operatorname{IgG}$ when compared with IGIV infusion in patients with PID [44]. A total of 11 patients with PID were enrolled in the study; ten achieved monthly doses of $25.5-61.2 \mathrm{~g}$ in a single site at rates of $120-300 \mathrm{ml} / \mathrm{h}$. A dose of $\geq 50 \mathrm{U}$ $\mathrm{rHuPH} 20 / \mathrm{g}$ IgG was required to facilitate IgG infusions. One patient withdrew from the study owing to moderate discomfort with the 1-week dose. Among the ten patients who completed the study, no allergic reactions related to study drug were observed, and all local reactions (e.g., swelling and redness) were considered mild in severity. Results of this study showed that $\mathrm{rHuPH} 20$ enabled single-site sc. administration, with bioavailability comparable with that achieved with IGIV infusion.

\section{IGHy Phase III clinical studies in patients with PID}

Pivotal study 160603

Following completion of the pilot study, a prospective, open-label, noncontrolled Phase III study (study 160603; Clinicaltrials.gov identifier: NCT00814320) was conducted to evaluate the efficacy, safety, tolerability and PKs of IGHy in 87 patients (age range: 4-78 years) with PID [16]. All patients received IGIV every 3-4 weeks for 3 months (epoch 1 ) at the previous dose of IGIV or IGSC that had been found to control their infections. This was followed by treatment with IGHy for 14-18 months (epoch 2). To allow patients to become accustomed to the large volumes of sc. fluid, they started with a ramp-up period; patients started with a 1-week dose followed a week later with a 2 -week dose, and so on, until they reached the 3- or 4-week interval that was used prior to the study. For example, for those patients being treated on a 4-week IGIV schedule, the doses were $25,50,75$ and $100 \%$ of the iv. dose administered at intervals of 1,2 and 3 weeks, respectively, to reach their every-4-week target dosing interval. IGHy was administered at each patient's prestudy IGIV dose and frequency, and $\mathrm{rHuPH} 20$ was administered at a dose ratio of $75 \mathrm{U} / \mathrm{g}$ IgG, followed immediately by an IgG $10 \%$ infusion at $108 \%$ of the previous weekly equivalent IGIV dose through the same site (using a 24-gauge sc. needle). In total, 31 of the patients in this study had been previously treated with both IGIV and IGSC (without rHuPH20) in a Phase III trial [45] and were then immediately rolled over into the IGHy part of the Phase III study. IGIV and IGSC data were used to compare safety, tolerability and PK with IGHy. The primary efficacy end point of the Phase III study was, per the FDA guidance, the mean rate of validated acute serious bacterial infections (SBIs; includes bacteremia/sepsis, bacterial meningitis, osteomyelitis/septic arthritis, bacterial pneumonia and visceral abscesses that are caused by a recognized bacterial pathogen) per patient per year during the 12-month efficacy period (epoch 2, excluding ramp-up).

\section{Infusion parameters}

Overall, patients received a total of 1129 IGHy infusions, the majority (94\%) of which were administered using a single site (median infusion sites/month: 1.09 for IGHy compared with 21.43 for those patients who had previously participated in a study of $\operatorname{IgG}$ $10 \%$ administered weekly by sc. infusion without rHuPH20 (Table 2). A total of $97 \%$ of infusions were administered without interruption, discontinuation or reduction in flow rate. Changes in flow rate $(1.7 \%$ reduced, $0.4 \%$ interrupted and $0.2 \%$ stopped) were due to AEs or tolerability problems.

The mean volume per site of IGHy given on a 2-, 3- or 4-week treatment schedule was $292.2 \mathrm{ml}$. The median total time to complete the infusion was slightly shorter with IGHy $(2.08 \mathrm{~h}$, including $\mathrm{rHuPH} 20)$ than with IGIV $(2.33 \mathrm{~h})$. The median maximum infusion rate for a single site was $300 \mathrm{ml} / \mathrm{h}$ (range: $10-300 \mathrm{ml} / \mathrm{h}$ ) for IGHy and $200 \mathrm{ml} / \mathrm{h}$ (range: $25-668 \mathrm{ml} / \mathrm{h}$ ) for IGIV (see Table 2). 
Table 2. Infusion parameters in the Phase III study in patients with primary immunodeficiencies (study 160603; Clinicaltrials.gov identifier: NCT00814320).

\begin{tabular}{|c|c|c|c|}
\hline Parameter & IGHy & IGIV & IGSC \\
\hline $\begin{array}{l}\text { Duration of IgG infusion }{ }^{\dagger}, \mathrm{h}, \text { median } \\
\text { (min-max) }\end{array}$ & $2.08(0.83-4.68)(n=81)$ & $2.33(0.92-6.33)(n=87)$ & NA \\
\hline $\begin{array}{l}\text { Maximum infusion rate of } \mathrm{lg}, \mathrm{ml} / \mathrm{h}, \\
\text { median (min-max) }\end{array}$ & $300(10-300)(n=81)$ & $200(25-668)(n=87)$ & NA \\
\hline Infusion volume per site, range & $91-648[46]$ & $75-800$ & NA \\
\hline $\begin{array}{l}\text { Number of infusion sites per month, } \\
\text { median (min-max): } \\
\text { - Study } \operatorname{arm} 1^{\ddagger} \\
\text { - Study } \operatorname{arm} 2\end{array}$ & $\begin{array}{l}1.09(1.0-3.5)(n=30) \\
1.09(0.8-4.3)(n=51)\end{array}$ & $\begin{array}{l}1.09(1.1-1.4)(n=31) \\
1.09(0.4-1.5)(n=56)\end{array}$ & $\begin{array}{l}21.43(9.0-44.3)(n=31) \\
\text { NA }\end{array}$ \\
\hline $\begin{array}{l}\text { Total number of infusions } \\
\text { - No reduction, interruption or } \\
\text { stoppage of infusion, } \mathrm{n}(\%)\end{array}$ & $\begin{array}{l}1129(\mathrm{n}=81) \\
1103(97.7)\end{array}$ & $\begin{array}{l}365(n=87) \\
350(95.9)\end{array}$ & $\begin{array}{l}\text { NA } \\
\text { NA }\end{array}$ \\
\hline \multicolumn{4}{|c|}{$\begin{array}{l}\text { †Defined as a time interval between start of rHuPH2O infusion and stoppage of IgG infusion. } \\
\text { 'Included patients who had also received IGSC in a previous study; data from the prior study were used in the present analysis. } \\
\text { IGHy: Recombinant human hyaluronidase-facilitated subcutaneous infusion of IgG; IGIV: Intravenous infusion of IgG; IGSC: Subcutaneous infusion of IgG; } \\
\text { max: Maximum; min: Minimum; NA: Not applicable; rHuPH20: Recombinant human hyaluronidase. } \\
\text { Data taken from [16]. }\end{array}$} \\
\hline
\end{tabular}

\section{Efficacy}

During treatment with IGHy (12-month efficacy period), two acute SBIs (both had bacterial pneumonia and were treated as outpatients using oral antibiotics) were reported (rate $=0.025$ [upper 95\% CI limit, 0.046] per patient-year). In addition, one case of bacterial pneumonia was reported during the ramp-up period. This is similar to what was previously observed in treatment with IGIV and IGSC [7,45,47,48]. The annual rate of any infection in patients receiving IGHy during the 12-month efficacy phase versus 3 months of IGIV treatment was 2.97 (95\% CI: 2.51-3.47) and 4.51 (95\% CI: 3.50-5.69), respectively. No serious AEs related to treatment with IGHy were reported.

\section{Tolerability}

Typically, the most frequent AEs with sc. infusions are local, whereas systemic AEs are more frequent in association with iv. infusions. Local AEs, regardless of cause, occurred at a rate of 0.199 per IGHy infusion and 0.011 per IGIV infusion (total IGIV infusions $=365$ ). The most common local AEs were pain/discomfort, erythema, swelling/edema (Figure 4) and pruritus. The majority were mild to moderate in severity; three were considered severe (infusion-site pain, infusion-site swelling and genital edema), with no sequelae. Local AEs related to treatment with IGHy occurred at a rate of 0.203 per infusion (comparable with published AE rates $[45,49,50]$ in studies of conventional weekly IGSC administration).

Systemic AEs (occurring within $72 \mathrm{~h}$ of infusion) occurred less frequently with IGHy treatment $(8.3 \%$ of infusions; 95\% CI: 6.3-10.0) than with IGIV treatment
(25\% of infusions; 95\% CI: 16.7-25.0). The most frequent systemic AEs, regardless of cause, were headache, fatigue, nausea, pyrexia, vomiting and chills (Table 3 ).

The rate of both local and systemic adverse drug reactions (ADRs) decreased over the course of this study, as well as during the extension study. This is discussed in more detail in the following paragraph.

In total, 13 out of $83(15.7 \%)$ patients exposed to IGHy developed antibodies capable of binding rHuPH20. However, there was no correlation with occurrence of local or systemic AEs. No patients developed neutralizing antibodies against $\mathrm{rHuPH} 20$.

\section{Quality of life}

Of the 69 patients who completed a questionnaire on treatment preference, the majority $(83 \%)$ indicated that they would prefer to continue receiving treatment with IGHy rather than to receive treatment with IGSC or IGIV. Patients indicated that they 'liked' or 'liked very much' the overall convenience $(86 \%)$, the ability to fit treatment into their schedules $(83 \%)$ and the administration frequency $(77 \%)$ of IGHy.

\section{Pharmacokinetics}

The bioavailability of IGHy (as measured by the area under the concentration vs time curve [AUC]) was 93.3\% (90\% CI: 91.4-95.2) of IGIV, which is within the margins of $\mathrm{PK}$ equivalence, defined as $80-125 \%$ by the FDA [51]. IGHy also showed improved bioavailability over IGSC, with a $20.4 \%$ improvement when the sc. infusion was facilitated by $\mathrm{rHuPH} 20$.

Consistent with slower absorption into the intravascular compartment, median peak serum IgG 


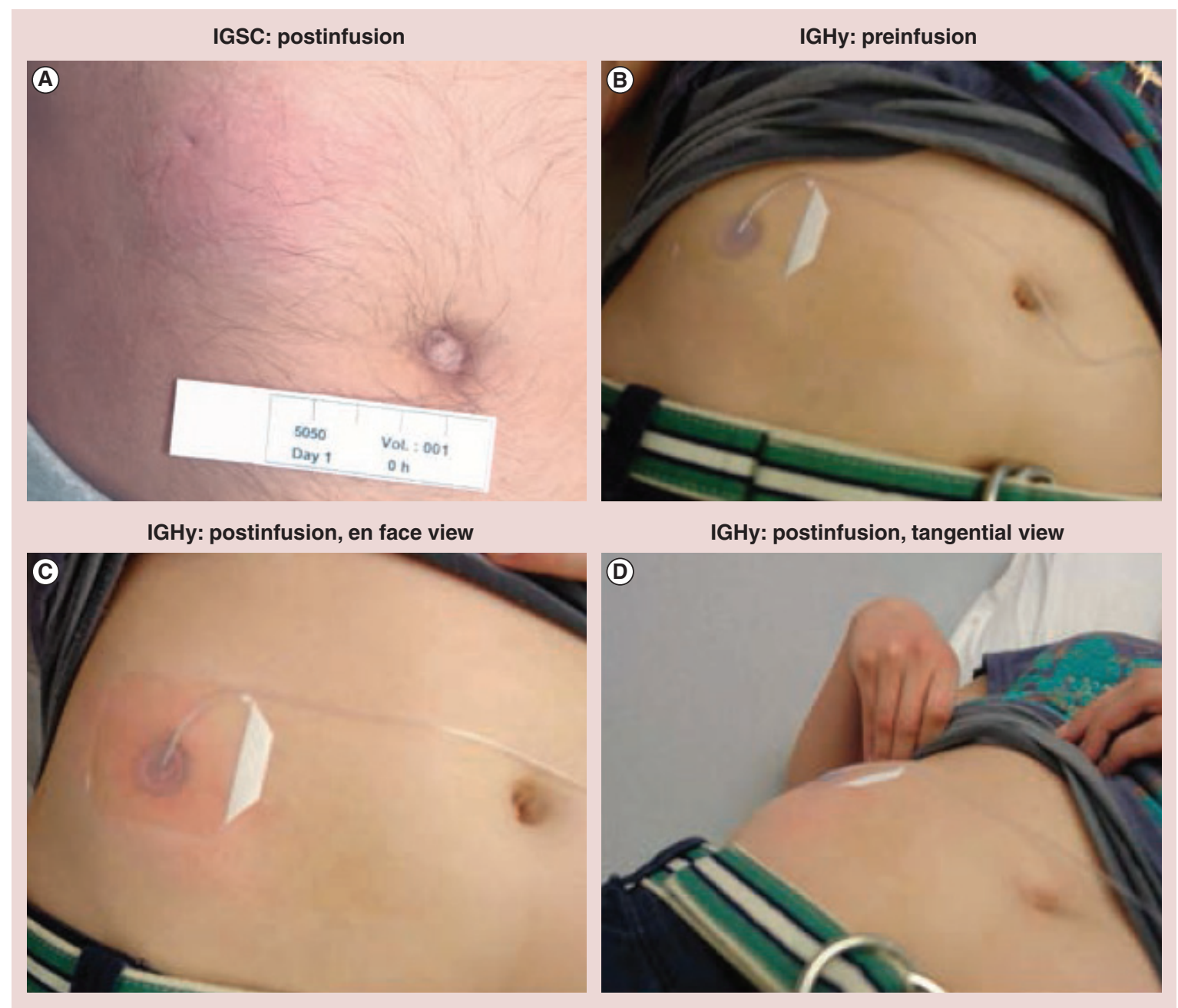

Figure 4. Reaction to subcutaneous infusion of IgG versus recombinant human hyaluronidase-facilitated subcutaneous infusion of IgG $^{+}$. (A) An IGSC site immediately after a 15-ml infusion. (B) The baseline appearance of the abdomen of a patient after placement of a subcutaneous catheter prior to infusion. (C) The en face view of the patient's abdomen immediately after the infusion of $300 \mathrm{ml}$ of $10 \% \mathrm{lg}$ preceded by infusion of recombinant human hyaluronidase $(\mathrm{rHuPH} 20)$. Note the mild erythema that is approximately $10 \mathrm{~cm}$ in diameter. (D) The tangential view of the patient's abdomen showing induration raising the skin surface approximately $1 \mathrm{~cm}$ immediately after the infusion of $300 \mathrm{ml}$ of $10 \% \mathrm{lgG}$ preceded by infusion of rHuPH 20.

${ }^{+}$Delivery of IGHy is associated with a diffuse bump at the injection site, which may last 1-3 days. The size of the bump varies depending on the volume administered. In this patient, approximately $300 \mathrm{ml} \mathrm{lgG} 10 \%$ plus rHuPH20 was infused.

IGHy: Recombinant human hyaluronidase-facilitated subcutaneous infusion of IgG; IGSC: Subcutaneous infusion of IgG.

Reproduced with kind permission from (A) CSL Behring; (B-D) Richard L Wasserman (DallasAllergylmmunology, TX, USA).

levels $\left(\mathrm{C}_{\max }\right)$ were lower with IGHy $(15.5 \mathrm{~g} / \mathrm{l})$ than with $\operatorname{IGIV}(21.9 \mathrm{~g} / \mathrm{l})$; slower absorption is indicated by the later peak $\mathrm{IgG}$ concentration $\left(\mathrm{T}_{\max }\right)$, which occurred a median of 5 days after infusion with IGHy (compared with 0.1 days following IGIV infusion).

Differences in serum IgG trough levels reflect differences in bioavailability when $\operatorname{IgG}$ is dosed at the same frequency either iv. or sc. When IGHy or IGIV was administered at 3- or 4-week treatment intervals, serum IgG trough levels were similar, regardless of the administration route (IGHy or IGIV) or patient age (Figure 5). The median ratio of serum IgG trough levels for IGHy/IGIV was $103.8 \%$ for patients aged $2-11$ years and $98.5 \%$ for patients aged $\geq 12$ years. The bioavailability of IGHy (measured by the AUC) was 93.3\% of IGIV, which indicated PK equivalence.

\section{Study conclusion}

The Phase III study showed that $\mathrm{rHuPH} 20$ permitted infusion of a 3- or 4-week IgG dose in a single site 
Table 3. Temporally associated systemic adverse events, regardless of causality, occurring in $>5 \%$ of patients during treatment with recombinant human hyaluronidase-facilitated subcutaneous infusion of IgG and intravenous infusion of IgG in the efficacy period.

\begin{tabular}{|c|c|c|c|c|}
\hline \multirow[t]{2}{*}{$\mathrm{AE}$} & \multicolumn{2}{|c|}{ IGHy $(n=81)$} & \multicolumn{2}{|c|}{ IGIV $(n=87)$} \\
\hline & Patients (\%) & $\mathrm{AE}$ rate/1000 infusions ${ }^{\dagger \neq}$ & Patients (\%) & AE rate/1000 infusions ${ }^{\ddagger}$ \\
\hline Headache & 19.8 & 31 & 25.3 & 112 \\
\hline Fatigue & 8.6 & 12 & 9.2 & 27 \\
\hline Nausea & 7.4 & 10 & 11.5 & 27 \\
\hline Pyrexia & 7.4 & 9 & 5.7 & 16 \\
\hline Vomiting & 7.4 & 8 & 5.7 & 16 \\
\hline Chills & 1.2 & 3 & 8.0 & 25 \\
\hline \multicolumn{5}{|c|}{$\begin{array}{l}{ }^{\dagger} \text { A total of } 1129 \text { IGHy infusions. } \\
{ }^{*} \text { A total of } 365 \text { IGIV infusions. } \\
\text { AE: Adverse event; IGHy: Recombinant human hyaluronidase-facilitated subcutaneous infusion of IgG; IGIV: Intravenous infusion of IgG. } \\
\text { Adapted with permission from [16] Elsevier. }\end{array}$} \\
\hline
\end{tabular}

in most patients, with infusion rates and bioavailability comparable with IGIV. Rates of acute SBIs in patients treated with IGHy were not different than rates observed in patients treated with IGIV or IGSC, and IGHy was well tolerated at infusion volumes, intervals and rates equivalent to the patients' previous IGIV administrations. Most patients also preferred IGHy over IGSC and IGIV, citing issues related to quality of life. Administered at the same 3- to 4-week dosing intervals as IGIV, IGHy provided bioavailability (determined by the AUC) similar to that of IGIV administration with a smaller dose adjustment than is recommended for IGSC.

The favorable results of this study supported the approval of IGHy in 2013 by the EMA for use in Europe as replacement therapy in patients aged $\geq 18$ years with PIDs, or myeloma or chronic lymphocytic leukemia with severe secondary hypogammaglobulinemia and recurrent infections [29].

\section{Extension study: 160902}

Study 160902 (Clinicaltrials.gov identifier: NCT01175213) was an extension of the Phase III study and was designed to collect long-term safety data on patients exposed to up to 5 years of IGHy treatment [52]. A total of 66 out of the 68 patients who completed the Phase III study were enrolled into the extension study at their prestudy IGHy dose and frequency of every 3-4 weeks. Following 3 months of treatment with IGHy, some patients were voluntarily switched to a 2 -week dosing interval to evaluate effects on $\operatorname{IgG}$ trough levels. Although there were no clinical safety signals regarding the use of IGHy, exposure to the rHuPH20 component of IGHy was discontinued after approximately 3 years, in July 2012, due to an FDA request for additional preclinical data on the potential impact of antibodies that bind $\mathrm{rHuPH} 20$. Patients were followed for an additional 24-48 weeks while receiving iv. or conventional sc. treatment.

At the conclusion of the study in August 2013, 49 patients had completed the extension study, with cumulative exposure to IGHy of up to 188 weeks and

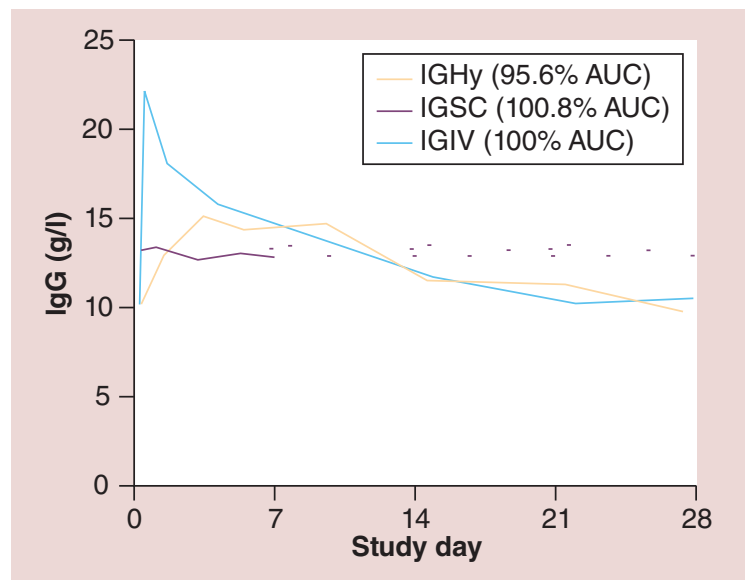

Figure 5. Serum IgG concentration for recombinant human hyaluronidase-facilitated subcutaneous infusion of IgG compared with intravenous infusion of IgG and subcutaneous infusion of $\mathrm{IgG}^{+}$in one representative patient with primary immunodeficiencies (study 160603).

${ }^{\dagger}$ Representative pharmacokinetic curves for one patient in the Phase III study (study 160603) comparing a 4-week infusion of IGIV, a weekly infusion of IGSC $10 \%$ at $143 \%$ of the intravenous dose with the same data points extended across the 4-week period to facilitate comparison with the other curves and a 4-week infusion of IGHy at $104 \%$ of the intravenous dose. AUC: Area under the concentration versus time curve; IGHy: Recombinant human hyaluronidase-facilitated subcutaneous infusion of IgG; IGIV: Intravenous infusion of IgG; IGSC: Subcutaneous infusion of IgG. Reproduced with permission from [16] Elsevier. 
187.69 patient-years of exposure to $\mathrm{rHuPH} 20$ [Baxter, Data on File]. No patients have withdrawn owing to a drug-related reaction, and no serious drug-related events were reported. The annual infection rate $(2.86$ per patient-year [95\% CI: 2.36-3.43]) was similar to that observed in the Phase III study and was stable or decreased over the course of the two studies. The overall rate throughout both the initial Phase III and extension studies was 2.99 infections per patient-year (95\% CI: 2.60-3.92); the rate declined from 3.25 in the first year to 2.33 in the third year. The rate of local and systemic ADRs per infusion in the extension study was 0.103 and 0.159 , respectively, which are lower than during the pivotal trial. Overall, the rate per infusion of both local and systemic ADRs declined each year of the combined studies (Table 4). There were no clinically observable changes in the skin or sc. tissue after 2959 IGHy infusions (including ramp-up in the initial Phase III study) [Baxter, Data on File] and 61 infusions in the Phase I/II study [53].

In the combined studies, after the ramp-up, there were 2729 IGHy infusions, and the majority (97.8\%) of patients required no changes in administration, including rate reduction, interruption or discontinuation as a result of tolerability concerns or AEs. Reducing the dosing interval from 4 to 2 weeks (at the same monthly dose) resulted in a mean increase of $13 \%$ in serum IgG trough levels. The $13 \%$ increase is similar to the increase of $15 \%$ over iv. troughs predicted by modeling for a sc. product given every 2 weeks [54]. However, the HyQvia troughs were achieved with a dose increase of $108 \%$ (vs iv.) rather than the $153 \%$ used for the sc. product. In total, 13 out of 66 patients had at least one non-neutralizing anti-rHuPH20 antibody titer $\geq 1: 160$; however, similar to observations in the Phase III study, there was no association with any local or systemic AEs, and no patients had neutralizing anti-rHuPH20 antibodies. Two additional patients developed a single positive $\mathrm{rHuPH} 20$ binding antibody titer of 1:160 during the extension study, but there was no association with any local or systemic AEs [Baxter, Data on File]. Following the discontinuation of IGHy, no rHuPH20-associated AEs were observed at follow-up. The study was completed in August 2013.

\section{Discussion}

There have been significant improvements in the formulation and administration of IgG therapy since the first patient was treated more than 60 years ago with concentrated human serum IgG administered subcutaneously [55]. These advances have significantly impacted the lives of patients who may require lifelong IgG treatment for PID. Health-related quality of life is improved in patients when they are treated with $\mathrm{IgG}$ administered subcutaneously compared with iv. administration [7,56]. Formulations that can be administered subcutaneously enable self-administration in a homebased setting, without the necessity for regular travel to a doctor's office, infusion center or clinic to receive treatment. The need for iv. access, which usually requires the assistance of a healthcare professional, is avoided. Rates of systemic AEs are also lower with sc. administration compared with iv. administration.

Standard IGSC administration, however, has its drawbacks. By virtue of the ECM barrier to bulk fluid flow, it is difficult to infuse large volumes of $\operatorname{IgG}$ into the sc. site. IgG volumes, when administered by the sc. route, currently range from 15 to $30 \mathrm{ml}$ per injection site; therefore, multiple infusion sites (weekly or biweekly) are required to attain a therapeutic dose [44,57-60]. In addition, the lower bioavailability of IGSC necessitates increasing the dose to between 137 and $153 \%$ of the IGIV dose to attain equivalent systemic IgG exposure, as determined by the AUC $[45,49,61]$. Clinical study data show that there is a correlation between increased serum IgG concentrations with a lower risk of serious infections, such as pneumonia [62]. Another recent analysis of data [63] from two studies of sc. administration with different doses of an $\operatorname{IgG} 20 \%$ formulation (mean $807.2 \mathrm{mg} / \mathrm{kg} / \mathrm{month}$ or $474.8 \mathrm{mg} / \mathrm{kg} / \mathrm{month}$ ) indicated that patients treated with a higher $\mathrm{IgG}$ dose spent less time in the hospital ( 0.2 vs 3.48 days) and missed fewer days of work or school (2.06 vs 8.00 days per patient-year) than patients treated with the lower (mean $474.8 \mathrm{mg} / \mathrm{kg} / \mathrm{month}$ ) dose [64,65]. These two studies, however, were not powered to detect a relationship between dose and outcome; furthermore, as one study was conducted in the USA, where higher doses are generally used [64], and the other in Europe, [65]

Table 4. Changes in the rate of adverse drug reactions per patient-year under recombinant human hyaluronidase-facilitated subcutaneous infusion of IgG treatment.

\begin{tabular}{|lllll|}
\hline \multirow{2}{*}{ Type of ADR } & \multicolumn{4}{c|}{ Changes in the rate of ADRs per patient-year } \\
\cline { 2 - 5 } & Year 1 (months 1-12) & Year 2 (months 13-24) & Year 3 (months 25-36) & Total for entire treatment period \\
Local & 3.68 & 2.12 & 0.37 & 2.60 \\
\hline Systemic & 1.94 & 1.24 & 0.63 & 1.75 \\
\hline $\begin{array}{l}\text { ADR: Adverse drug reaction. } \\
\text { Data taken from [BAXTER, DATA ON FILE]. }\end{array}$ & & & \\
\hline
\end{tabular}


potential differences in healthcare utilization must be taken into account [63].

Use of $\mathrm{rHuPH} 20$ to facilitate the absorption and dispersion of products other than IgG has, for example, been shown to enhance insulin delivery in healthy volunteers and patients with diabetes Type 1 or 2 , with a concomitant improvement in glycemic control, a reduction in risk of hypoglycemia and a reduction in intrapatient variability in insulin absorption/ action over time [66]. Similarly, the use of rHuPH20 to facilitate IGSC infusion has also been studied in Phase III trials of patients with PID. Results from the pivotal Phase III study in patients with PID showed that, with IGHy, the majority of patients could receive full doses of $\mathrm{IgG}$ when administered every 3-4 weeks using a single sc. site without local or systemic tolerability concerns. IGHy was well tolerated at infusion volumes, intervals and rates equivalent to those associated with the patients' previous IGIV doses. No tolerability concerns were identified during the time period of up to 3 years of treatment with IGHy in the extension study, and the rate of both local and systemic ADRs declined over time (see Table 4). No clinically observable changes were noted in the skin or sc. tissue over the 3-year period of exposure. Safety and tolerability beyond 3 years of IGHy exposure are likely to be determined in postmarketing studies.

\section{Conclusion}

The use of IGHy for the treatment of patients with PID provides the combined benefits of both sc. and iv. administration. Similar to sc. administration, patients are able to self-infuse IGHy in a home-based setting without a requirement for iv. access and with a reduced risk for systemic AEs compared with IGIV. Similar to the benefit of iv. administration, the administration of IGHy enables patients to reduce dosing frequency to every 3-4 weeks compared with weekly or biweekly with sc. administration. By combining the benefits of iv. and sc. administration, options for patients in need of IgG treatment are increased with the further benefit of customizing IgG replacement therapy to meet individual patients' needs.

IGHy has great potential for dosing flexibility and may be administered as infrequently as once every 4 weeks or as often as the patient chooses to achieve an appropriate dose. Although the data are insufficient to make accurate projections, it is reasonable to expect that IGHy may be associated with fewer serious systemic AEs than IGIV. It is likely that, owing to self-administration, dosing flexibility and the possibility of fewer side effects than IGIV, many clinicians and patients will prefer IGHy. Baxter Healthcare Corporation, the manufacturer of IGHy, is currently conducting a Phase II/III trial (Clinicaltrials.gov identifier: NCT01218438) of an IGSC 20\% formulation for patients with PID, raising the possibility of an IGHy $20 \%$ formulation that would decrease infusion volumes and durations, thereby further increasing the options for patients.

Other foreseeable developments include formulating IgG and $\mathrm{rHuPH} 20$ in the same solution and clinical trials to support extending the use of IGHy to the immunomodulatory uses of IgG. The advantages of IGHy would be particularly important to those patients owing to their increased risk for serious systemic AEs.

Although not yet fully laid to rest, concerns about AEs due to anti-rHuPH20 antibody production have not borne out during studies of up to 3 years of exposure. The major remaining question is whether repeated disruption of the sc. space over time will result in irreversible injury to the sc. tissues, although no long-term effects of the forceful injection of 16 and $20 \%$ solutions of IgG without rHuPH20 have been observed over decades of use. This question will only be answered by postmarketing studies of very long duration.

The use of IGHy, after an introductory lag phase, can be expected to experience steadily increasing growth over the next 5-10 years owing to the features of the drug that are attractive to patients and clinicians, increasing identification of PID patients and data supporting the use of higher doses. For these reasons, IGHy may become the preferred mode of IgG therapy.

\section{Open Access}

This work is licensed under the Creative Commons Attribution-NonCommercial 3.0 Unported License. To view a copy of this license, visit http://creativecommons.org/licenses/bync-nd/3.0/

\section{Acknowledgements}

The author would like to thank Halozyme Therapeutics and CSL Behring for their generosity in providing Figure 3 and Figure 4A, respectively.

\section{Financial \& competing interests disclosure}

RL Wasserman has served as an investigator, consultant and speaker for Baxter International, CSL Behring; an investigator and consultant for ADMA, Biotest and Kedrion; an investigator for Korean Green Cross; and as a consultant for BPL. The author has no other relevant affiliations or financial involvement with any organization or entity with a financial interest in or financial conflict with the subject matter or materials discussed in the manuscript apart from those disclosed.

Baxter Healthcare Corporation provided funding for editorial support. RL Wasserman received no compensation related to the development of this manuscript, is fully responsible for all content and editorial decisions, and meets criteria for 
authorship as recommended by the International Committee of Medical Journal Editors. Writing and editorial assistance were provided by Penny Baron, MS, CMPP, of BlueMomen- tum, a division of KnowledgePoint360 Group, and Irene Durham, CMPP, previously employed at ReSearch Pharmaceutical Services, Inc.

\section{Executive summary}

Primary immunodeficiencies

- Patients with primary immunodeficiencies are susceptible to severe, recurrent infections.

- The standard of care is IgG replacement therapy via intravenous (iv.) or subcutaneous (sc.) administration.

- The iv. administration of IgG (IGIV) is burdensome and is also associated with a significant rate of systemic adverse events.

- The administration of sc. immunoglobulin (IGSC) is as effective as IGIV administration with fewer systemic adverse events, and infusions may be given at home; however, due to the extracellular matrix restriction of fluid flow, there is a limit to the amount of IgG that can be infused.

Hyaluronidase

- Transiently digesting extracellular matrix hyaluronan permits the conversion of drug therapies from iv. to sc. infusion.

- Hyaluronidase is indicated for the dispersion and absorption of other drugs and fluids.

- Animal-derived hyaluronidase products are often immunogenic in humans and can elicit IgE-mediated allergic reactions after repeated administration.

Recombinant human hyaluronidase

- Recombinant human hyaluronidase ( $\mathrm{rHuPH} 20)$ is fast acting, with a local half-life of less than $30 \mathrm{~min}$, and is undetectable in the plasma following sc. infusion.

- rHuPH20 enables sc. administration of large IgG volumes at a single site.

- The safety and efficacy of rHuPH2O in enhancing dispersion and absorption of fluids and drugs administered sc. has been demonstrated in clinical trials.

- $\mathrm{rHuPH} 20$ is approved for use in the USA and other countries.

rHuPH20-facilitated subcutaneous infusion of IgG in primary immunodeficiencies

- In most patients, rHuPH20 permitted infusion of a 3- or 4-week IgG dose in a single site.

- Infusion rates and bioavailability were comparable with those of IGIV.

- Bioavailability was better than with sc. administration.

- Acute serious bacterial infections rates did not differ between patients treated with rHuPH20-facilitated subcutaneous infusion of IgG (IGHy) or IGIV or IGSC.

- IGHy was well tolerated at infusion volumes, intervals and rates equivalent to the patients' previous IGIV administration.

- The majority of patients preferred IGHy over IGSC and IGIV.

\section{References}

Papers of special note have been highlighted as:

- of interest; $\bullet$ of considerable interest

1 International Union of Immunological Societies Expert Committee on Primary Immunodeficiencies, Notarangelo LD, Fischer A et al. Primary immunodeficiencies: 2009 update. J. Allergy Clin. Immunol. 124(6), 1161-1178 (2009).

2 Bousfiha AA, Jeddane L, Ailal F et al. Primary immunodeficiency diseases worldwide: more common than generally thought. J. Clin. Immunol. 33(1), 1-7 (2013).

3 Boyle JM, Buckley RH. Population prevalence of diagnosed primary immunodeficiency diseases in the United States. J. Clin. Immunol. 27(5), 497-502 (2007).

4 Wasserman RL. Progress in gammaglobulin therapy for immunodeficiency: from subcutaneous to intravenous infusions and back again. J. Clin. Immunol. 32(6), 1153-1164 (2012).

- Reviews IgG replacement therapy, including recombinant human hyaluronidase ( $\mathrm{rHuPH} 20)$-facilitated subcutaneous infusion of IgG (IGHy) in patients with primary immunodeficiencies (PIDs).

5 Berger M. Subcutaneous immunoglobulin replacement in primary immunodeficiencies. Clin. Immunol. 112(1), 1-7 (2004).

6 Bonilla FA. Intravenous immunoglobulin: adverse reactions and management. J. Allergy Clin. Immunol. 122(6), 1238-1239 (2008).

7 Berger M, Murphy E, Riley P, Bergman GE; VIRTUE Trial Investigators. Improved quality of life, immunoglobulin $\mathrm{G}$ levels, and infection rates in patients with primary immunodeficiency diseases during self-treatment with subcutaneous immunoglobulin G. South Med. J. 103(9), 856-863 (2010).

8 Daly PB, Evans JH, Kobayashi RH et al. Home-based immunoglobulin infusion therapy: quality of life and patient health perceptions. Ann. Allergy 67(5), 504-510 (1991).

9 Gardulf A, Andersen V, Björkander J et al. Subcutaneous immunoglobulin replacement in patients with primary 
antibody deficiencies: safety and costs. Lancet 345(8946), 365-369 (1995).

10 Gardulf A, Björvell H, Andersen V et al. Lifelong treatment with gammaglobulin for primary antibody deficiencies: the patients' experiences of subcutaneous self-infusions and home therapy. J. Adv. Nurs. 21(5), 917-927 (1995).

11 Gardulf A, Björvell H, Gustafson R, Hammarström L, Smith CI. The life situations of patients with primary antibody deficiency untreated or treated with subcutaneous gammaglobulin infusions. Clin. Exp. Immunol. 92(2), 200-204 (1993).

12 Gardulf A, Nicolay U, Math D et al. Children and adults with primary antibody deficiencies gain quality of life by subcutaneous IgG self-infusions at home. J. Allergy Clin. Immunol. 114(4), 936-942 (2004).

13 Gaspar J, Gerritsen B, Jones A. Immunoglobulin replacement treatment by rapid subcutaneous infusion. Arch. Dis. Child. 79 (1), 48-51 (1998).

14 Kittner JM, Grimbacher B, Wulff W, Jäger B, Schmidt RE. Patients' attitude to subcutaneous immunoglobulin substitution as home therapy. J. Clin. Immunol. 26(4), 400-405 (2006).

15 Nicolay U, Kiessling P, Berger M et al. Health-related quality of life and treatment satisfaction in North American patients with primary immunodeficiency diseases receiving subcutaneous IgG self-infusions at home. J. Clin. Immunol. 26(1), 65-72 (2006).

- Data demonstrate improved quality of life and treatment satisfaction in patients treated with home-based administration of subcutaneous immunoglobulin selfinfusion compared with treatment with intravenous administration of $\operatorname{IgG}$ in the home or doctor's office.

Wasserman RL, Melamed I, Stein MR et al. Recombinant human hyaluronidase-facilitated subcutaneous infusion of human immunoglobulins for primary immunodeficiency. J. Allergy Clin. Immunol. 130(4), 951-957 (2012).

-. Investigators provide the final results of the pivotal Phase III study of IGHy in patients with PIDs.

17 Meth MJ, Rosenthal DW, Bonagura VR. Subcutaneous immunoglobulin infusion to treat infants and toddlers with antibody deficiencies. Ann. Allergy Asthma Immunol. 105(2), 187-188 (2010).

18 Frost GI. Recombinant human hyaluronidase (rHuPH20): an enabling platform for subcutaneous drug and fluid administration. Expert Opin. Drug Deliv. 4(4), 427-440 (2007).

-. Provides the rationale for the use of $\mathrm{rHuPH} 20$ and reviews the in vitro, preclinical and clinical development of $\mathrm{rHuPH} 20$.

19 Duran-Reynals F. The effect of extracts of certain organs from normal and immunized animals on the infecting power of vaccine virus. J. Exp. Med. 50 (3), 327-340 (1929).

20 Kreil G. Hyaluronidases - a group of neglected enzymes. Protein Sci. 4(9), 1666-1669 (1995).

21 Toole BP. Hyaluronan: from extracellular glue to pericellular cue. Nat. Rev. Cancer 4(7), 528-539 (2004).
22 Hascall VC, Laurent T. Hyaluronan: structure and physical properties. www.glycoforum.gr.jp/science/hyaluronan/HA01/HA01E. html

23 Toole BP. Hyaluronans. In: Proteoglycans: Structure, Biology and Molecular Interactions. Iozzo R (Ed.). Marcel Dekker, NY, USA, 61-92 (2000).

24 Csoka AB, Frost GI, Stern R. The six hyaluronidase-like genes in the human and mouse genomes. Matrix Biol. 20(8), 499-508 (2001).

25 Vitrase $^{\circledast}$, prescribing information. ISTA Pharmaceuticals, CA, USA (2012).

26 Amphadase ${ }^{\circledast}$, prescribing information. Amphastar Pharmaceuticals Inc., CA, USA (2012).

27 Hylenex $^{\circledR}$, prescribing information. Halozyme Therapeutics, Inc., CA, USA (2012).

28 Woodcock J. US FDA, Department of Health and Human Services. Follow-on protein products (26 March 2007). www.hhs.gov/asl/testify/2007/04/t20070326a.html

29 HyQvia $(100 \mathrm{mg} / \mathrm{ml}$ solution for infusion for subcutaneous use) summary of product characteristics. Baxter Innovations GmbH; Vienna, Austria; 2013. www.ema.europa.eu/docs/en_GB/document_library/ EPAR_-_Product_Information/human/002491/ WC500143851.pdf

30 Yocum RC, Sugarman B, Vaughn D, Vick A, Brunelle R, Frost G. Pharmacokinetics and glucodynamics of an insulin analog and regular insulin injected with recombinant human hyaluronidase: fast-acting insulins made faster. Presented at: American Diabetes Association 68th Scientific Sessions. San Francisco, CA, USA, 6-10 June 2008.

31 Silverstein SM, Greenbaum S, Stern R. Hyaluronidase in ophthalmology. J. Appl. Res. 12(1), 1-13 (2012). Bookbinder LH, Hofer A, Haller MF et al. A recombinant human enzyme for enhanced interstitial transport of therapeutics. J. Control. Release 114(2), 230-241 (2006).

33 Eberhart AH, Weiler CR, Erie JC. Angioedema related to the use of hyaluronidase in cataract surgery. Am. J. Ophthalmol. 138(1), 142-143 (2004).

34 Borchard K, Puy R, Nixon R. Hyaluronidase allergy: a rare cause of periorbital inflammation. Australas. J. Dermatol. 51(1), 49-51 (2010).

35 Kim TW, Lee JH, Yoon KB, Yoon DM. Allergic reactions to hyaluronidase in pain management. A report of three cases. Korean J. Anesthesiol. 60 (1), 57-59 (2011).

36 Thompson CB, Shepard HM, O'Connor PM et al. Enzymatic depletion of tumor hyaluronan induces antitumor responses in preclinical animal models. Mol. Cancer Ther. 9(11), 3052-3064 (2010).

37 Kang DW, Jadin L, Nekoroski T, Drake FH, Zepeda ML. Recombinant human hyaluronidase $\mathrm{PH} 20$ ( $\mathrm{rHuPH} 20$ ) facilitates subcutaneous infusions of large volumes of immunoglobulin in a swine model. Drug Deliv. Transl. Res. 2(4), 254-264 (2012).

38 Allen CH, Etzwiler LS, Miller MK et al. Recombinant human hyaluronidase-enabled subcutaneous pediatric rehydration. Pediatrics 124(5), e858-e867 (2009). 
39 Harb G, Lebel F, Battikha J, Thackara JW. Safety and pharmacokinetics of subcutaneous ceftriaxone administered with or without recombinant human hyaluronidase ( $\mathrm{rHuPH} 20)$ versus intravenous ceftriaxone administration in adult volunteers. Curr. Med. Res. Opin. 26(2), 279-288 (2010).

40 Thomas JR, Wallace MS, Yocum RC, Vaughn DE, Haller MF, Flament J. The INFUSE-Morphine study: use of recombinant human hyaluronidase ( $\mathrm{rHuPH} 20)$ to enhance the absorption of subcutaneously administered morphine in patients with advanced illness. J. Pain Symptom. Manage. 38(5), 663-672 (2009).

41 Halozyme Pipeline. www.halozyme.com/Products-And-Pipeline/Pipeline/default. aspx

42 Knight E, Carne E, Novak B et al. Self-administered hyaluronidase-facilitated subcutaneous immunoglobulin home therapy in a patient with primary immunodeficiency. J. Clin. Pathol. 63(9), 846-847 (2010).

43 Subcuvia $160 \mathrm{~g} / \mathrm{l}$ solution for injection (human normal immunoglobulin) summary of product characteristics; Baxter AG; Vienna, Austria; 2011. www.ecomm.baxter.com/ecatalog/loadResource.do?bid=54579

44 Misbah S, Sturzenegger MH, Borte M et al. Subcutaneous immunoglobulin: opportunities and outlook. Clin. Exp. Immunol. 158(Suppl. 1), 51-59 (2009).

45 Wasserman RL, Melamed I, Kobrynski L et al. Efficacy, safety, and pharmacokinetics of a $10 \%$ liquid immune globulin preparation (GAMMAGARD LIQUID, 10\%) administered subcutaneously in subjects with primary immunodeficiency disease. J. Clin. Immunol. 31(3), 323-331 (2011a).

46 Stein M, Wasserman RL, Melamed I et al. Tolerability/efficacy/ pharmacokinetics of facilitated-subcutaneous infusion of human immune globulin G, 10\%, and recombinant human hyaluronidase in adults with primary immunodeficiency: Phase 3 study. Presented at: First International Primary Immunodeficiencies Congress. Estoril, Portugal, 7-8 November 2013.

47 Church JA, Leibl H, Stein MR et al. Efficacy, safety and tolerability of a new $10 \%$ liquid intravenous immune globulin [IGIV $10 \%]$ in patients with primary immunodeficiency. J. Clin. Immunol. 26(4), 388-395 (2006).

48 Ochs HD, Gupta S, Kiessling P, Nicolay U, Berger M; Subcutaneous IgG Study Group. Safety and efficacy of selfadministered subcutaneous immunoglobulin in patients with primary immunodeficiency diseases. J. Clin. Immunol. 26(3), 265-273 (2006).

49 Wasserman RL, Irani AM, Tracy J et al. Pharmacokinetics and safety of subcutaneous immune globulin (human), 10\% caprylate/chromatography purified in patients with primary immunodeficiency disease. Clin. Exp. Immunol. 161(3), 518-526 (2010).

50 Hagan JB, Fasano MB, Spector S et al. Efficacy and safety of a new $20 \%$ immunoglobulin preparation for subcutaneous administration, IgPro20, in patients with primary immunodeficiency. J. Clin. Immunol. 30(5), 734-745 (2010).

51 US Food and Drug Administration. Guidance for Industry: bioavailability and bioequivalence studies for orally administered drug products - general considerations. Version 1, March 2003.
www.fda.gov/downloads/Drugs/

GuidanceComplianceRegulatoryInformation/Guidances/ UCM070124.pdf

52 Melamed I, Wasserman RL, Stein M et al. Long-term tolerability and safety of facilitated-subcutaneous infusion of human immune globulin $\mathrm{G}(\mathrm{IgG}), 10 \%$, and recombinant human hyaluronidase (rHuPH20) (IGHy): a Phase 3 extension study in patients with primary immunodeficiencies (PIs). Presented at: American Association of Asthma, Allergy and Immunology Annual Meeting. CA, USA, 28 February-4 March 2014.

- This meeting abstract provides outcomes of the open-label extension of the IGHy pivotal trial after up to 3 years of treatment with IGHy.

53 Schiff R, Wasserman RL, Stein M et al. Recombinant human hyaluronidase facilitates dispersion of subcutaneously administered Gammagard Liquid, enabling administration of full monthly dose in single site with improved bioavailability in immunodeficient patients. Presented at: 13th Biennial Meeting of the European Society for Immunodefiencies. 's-Hertogenbosch, The Netherlands, 16-19 October 2008.

54 Rojavin M, Landersdorfer C, Bexon M, Pfister M, Sidhu J. Switching from monthly intravenous to biweekly subcutaneous immunoglobulin: a pharmacokinetic modeling and simulation approach. Presented at: American Academy of Allergy, Asthma and Immunology Annual Meeting. TX, USA, 22-26 February 2013.

55 Bruton OC. Agammaglobulinemia. Pediatrics 9(6), 722-728 (1952).

56 Gardulf A. Immunoglobulin treatment for primary antibody deficiencies: advantages of the subcutaneous route. BioDrugs 21(2), 105-116 (2007).

57 Moore ML, Quinn JM. Subcutaneous immunoglobulin replacement therapy for primary antibody deficiency: advancements into the 21st century. Ann. Allergy Asthma Immunol. 101(2), 114-121; quiz 122-123 (2008).

58 Gustafson R, Gardulf A, Hansen S et al. Rapid subcutaneous immunoglobulin administration every second week results in high and stable serum immunoglobulin G levels in patients with primary antibody deficiencies. Clin. Exp. Immunol. 152(2), 274-279 (2008).

59 Gardulf A, Nicolay U, Asensio O et al. Rapid subcutaneous $\mathrm{IgG}$ replacement therapy is effective and safe in children and adults with primary immunodeficiencies - a prospective multi-national study. J. Clin. Immunol. 26(2), 177-185 (2006).

60 Jolles S, Sleasman JW. Subcutaneous immunoglobulin replacement therapy with Hizentra, the first $20 \%$ SCIG preparation: a practical approach. $A d v$. Ther. 28(7), 521-533 (2011).

61 Wasserman RL, Melamed I, Nelson RP Jr et al. Pharmacokinetics of subcutaneous IgPro20 in patients with primary immunodeficiency. Clin. Pharmacokinet. 50(6), 405-414 (2011b).

62 Orange JS, Grossman WJ, Navickis RJ, Wilkes MM. Impact of trough $\mathrm{IgG}$ on pneumonia incidence in primary immunodeficiency: a meta-analysis of clinical studies. Clin. Immunol. 137(1), 21-30 (2010). 
- This meta-analysis demonstrates decreased incidence of pneumonia with increased levels of $\operatorname{IgG}$ trough in patients with PIDs.

63 Orange JS, Belohradsky BH, Berger M et al. Evaluation of correlation between dose and clinical outcomes in subcutaneous immunoglobulin replacement therapy. Clin. Exp. Immunol. 169(2), 172-181 (2012).

64 Jolles S, Bernatowska E, de Gracia J et al. Efficacy and safety of Hizentra ${ }^{\circledR}$ in patients with primary immunodeficiency after a dose-equivalent switch from intravenous or subcutaneous replacement therapy. Clin. Immunol. 141(1), 90-102 (2011).
65 Maroto Hernando M, Soler Palacín P, Martin Nalda N, Oliveras Arenas M, Español Boren T, Figueras Nadal C. [Subcutaneous gammaglobulin in common variable immunodeficiency. First experience in Spain.] An. Pediatr. (Barc.) 70(2), 111-119 (2009).

66 Muchmore DB, Vaughn DE. Accelerating and improving the consistency of rapid-acting analog insulin absorption and action for both subcutaneous injection and continuous subcutaneous infusion using recombinant human hyaluronidase. J. Diabetes Sci. Technol. 6(4), 764-772 (2012). 